\title{
Family History of Coronary Artery Disease as an Additional Risk Factor Associated with Coronary Artery Disease: A Descriptive Observational Study
}

\author{
Prashanth Kulkarni* \\ Department of Cardiology, Heart Institutes, Apollo BGS hospitals, Mysore-570023, Karnataka, India
}

Received: November 07, 2014; Accepted: December 11, 2014; Published: January 10, 2015

*Corresponding author: Prashanth Kulkarni, Department of Cardiology, Heart Institutes, Apollo BGS hospitals, Mysore-570023, Karnataka, India, Email: docpk77@gmail.com

\begin{abstract}
Background: Globally Coronary Artery Disease (CAD) has emerged as one of the leading cause of death. This descriptive observational study was under taken to assess the role of family history of premature Coronary artery disease as an additional risk factor for $\mathrm{CAD}$

Methods and materials: Data were prospectively collected of 1000 consecutive patients of Ischemic heart disease who underwent cardiac catheterization and subsequent revascularisation at Heart institutes, Apollo BGS hospitals, Mysore, India between April 1, 2013, and March 31, 2014. Among these patients in addition to traditional risk factors for coronary artery disease like diabetes mellitus, obesity, hyperlipidemia, hypertension, smoking and obstructive sleep apnoea, the role of family history of premature coronary artery disease was assessed.
\end{abstract}

Results: Total number of patients who underwent cardiac catheterization during the study period was 1000 .Mean age was $62.2 \pm 11.4 y r s$. Male comprised 720 patients (72\%) and females 280 patients (28\%). Positive family history of premature CAD was present in $32 \%$ (320 patients) of the total CAD patients studied. When family history of premature CAD was considered as risk factor, the mean age of CAD diagnosis was at 56 years compared to 62.2 years for those with a negative family history of CAD.

Conclusion: Family history of premature CAD is an additional risk factor and lowers the age of diagnosis for CAD.

\section{Introduction}

Over the last decade, cardiovascular disease especially coronary heart disease has become the largest cause of death worldwide. By 2030, World Health Organization predicts that worldwide $14.9 \%$ of death in men and $13.1 \%$ of deaths in women will be caused by Coronary heart disease [1]. Family history of CAD is considered a major risk factor in disease development not only because of inherited susceptibility genes, but also because of shared lifestyles that may exacerbate individual susceptibility to $\mathrm{CAD}[2]$.

There are three ways in which a positive family history might be related to the development of coronary heart disease: (a) as an index of the inheritance of risk factors; (b) as a truly independent risk factor; and (c) as a vulnerability factor potentiating the action of risk factors [3].

The aim of this study was to determine the presence of family history of premature CAD and assess its impact on CAD risk, independent of established risk factors like diabetes mellitus, obesity, hyperlipidemia, hypertension, smoking and obstructive sleep apnoea.

\section{Methods}

\section{Study population}

This was a descriptive observational study. Data were prospectively collected of 1000 consecutive patients of Ischemic heart disease who underwent cardiac catheterization and subsequent revascularisation at Heart institutes, Apollo BGS hospitals Mysore, India between April 1, 2013, and March 31, 2014. Among these patients the presence of family history of premature CAD was noted by the commonly used definition of premature coronary artery disease that has been utilized in the Framingham study (family history of $<55$ years in men and $<65$ years in women, first degree relatives) and its impact on CAD risk was determined, controlling for diabetes mellitus, obesity, hyperlipidemia, hypertension, smoking and obstructive sleep apnoea. This study was approved by the Institutional Review Board and informed consent was obtained from all the patients who participated in the study.

\section{Data collection and analysis}

Patient, disease and procedure characteristics were collected prospectively on all patients in the study. Patient characteristics included age, gender, diabetes mellitus, hypertension, obesity, hyperlipidemia, smoking, obstructive sleep apnoea and family history of CAD. Cardiac disease characteristics included history of myocardial infarction and unstable angina. Cardiovascular procedure characteristics recorded were type of revascularisation undergone like Percutaneous Coronary Intervention (PCI) and Coronary Artery Bypass Graft (CABG). The data obtained was 
tabulated and analyzed using rates, ratios, percentages and chisquare test.

\section{Results}

The mean age was $62.2 \pm 11.4$ years. Males comprised 720 patients $(72 \%)$ and females 280 patients (28\%). Family history of premature CAD was present in 320 patients (32\%) of which males were 224 (70\%) and females were 96 (30\%). When family history of premature CAD was considered as risk factor for CAD the mean age of at CAD diagnosis was at 56 years, compared to 62.2 years for the negative family history of CAD patient category (Table 1). Family history of premature coronary artery disease during an encounter with the patient has greater predictive value and in this study it did affect the age of disease diagnosis which occurred earlier compared to overall study population.

\section{Discussion}

As observed in this study family history of premature CAD indicates the possible presence of deleterious mutations and this can make an individual susceptible for premature CAD. Therefore, the relationship between family history and disease risk might be used to separate population, behavioural, and environmental impacts of family history and help determine the genetic effect [4]. The INTERHEART study, an international case-control study, carried out in 52 countries concluded that abnormal lipids, smoking, hypertension, diabetes, abdominal 